\title{
Photochromic composite for random lasing based on porous polypropylene infiltrated with azobenzene-containing liquid crystalline mixture
}

\author{
Victor Lisinetskii $*^{1}$, Alexander Ryabchun ${ }^{2,3}$, Alexey Bobrovsky ${ }^{3}$, Sigurd Schrader ${ }^{1}$ \\ ${ }^{1}$ Technical University of Applied Sciences Wildau, Hochschulring 1, 15745 Wildau, Germany \\ ${ }^{2}$ Fraunhofer Institute for Applied Polymer Research, Geiselbergstr. 69, 14476 Potsdam-Golm, \\ Germany \\ ${ }^{3}$ Faculty of Chemistry, Moscow State University, Leninskie Gory, 119991 Moscow, Russia \\ *E-mail: lisinetskii@gmail.com, phone: (+49) 3375508461.
}




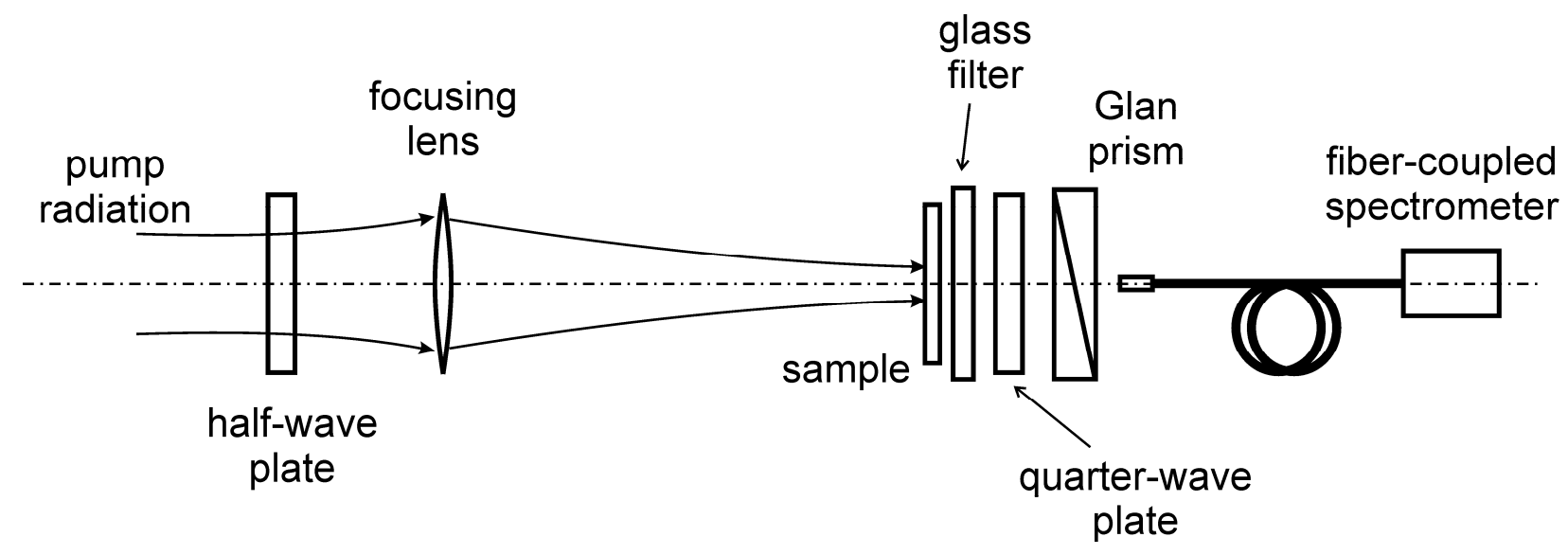

Figure S1. Scheme of the experimental setup.

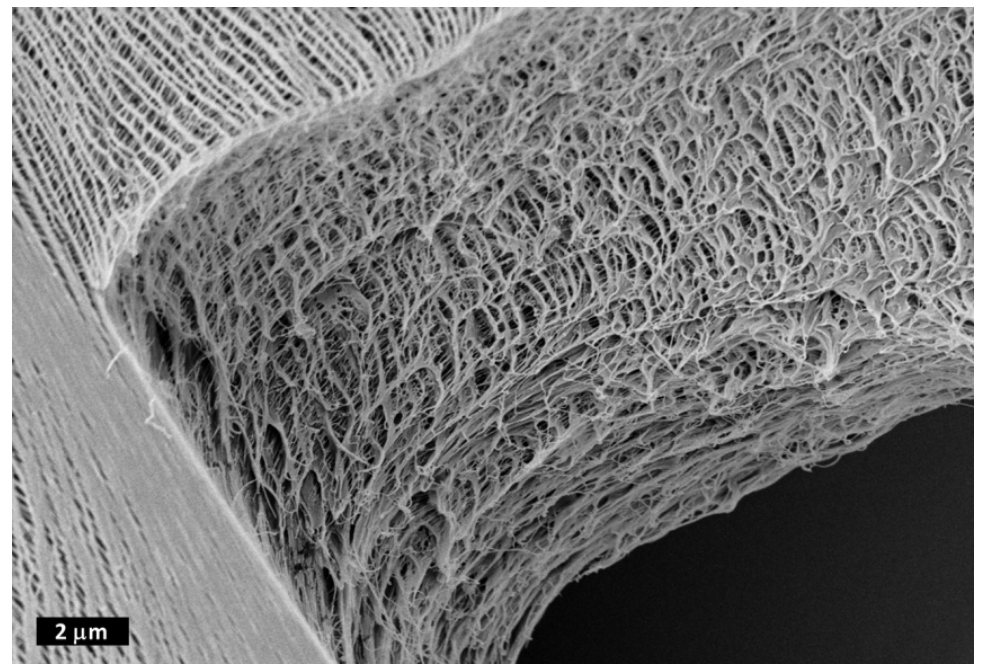

Figure S2. SEM image of the cross section of a PP porous film (Celgard 2500).

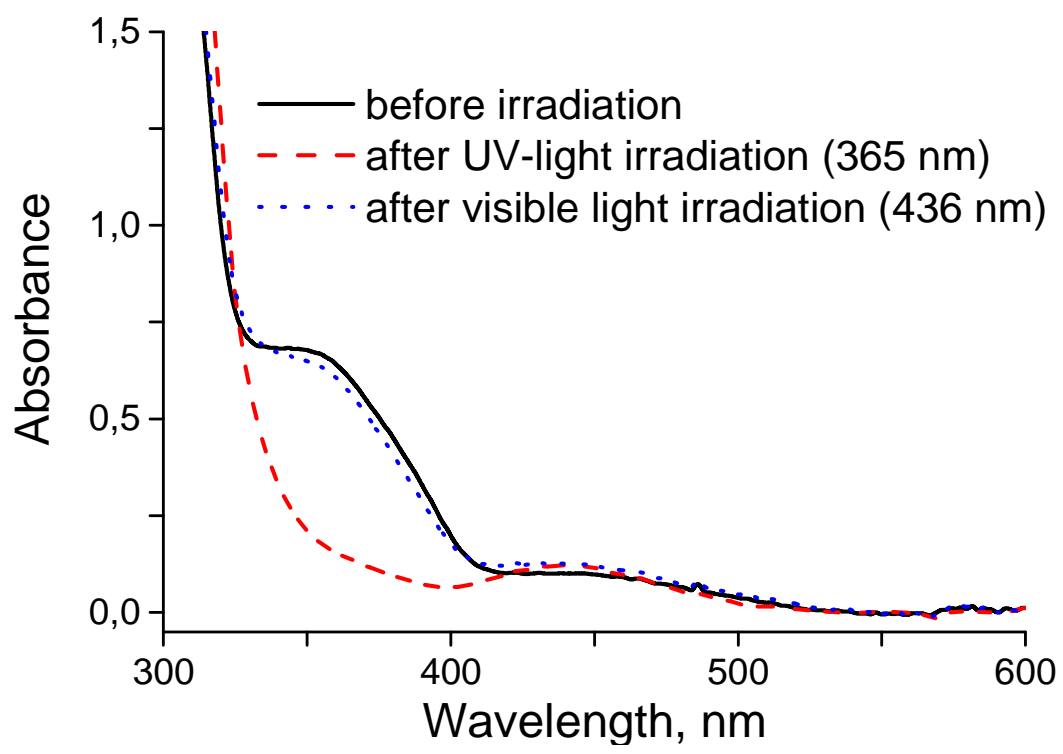

Figure S3. Change of absorbance spectrum of the mixture 5CB and 6DABU (70:30) after UV (red line) and followed visible light (blue line) exposure. 


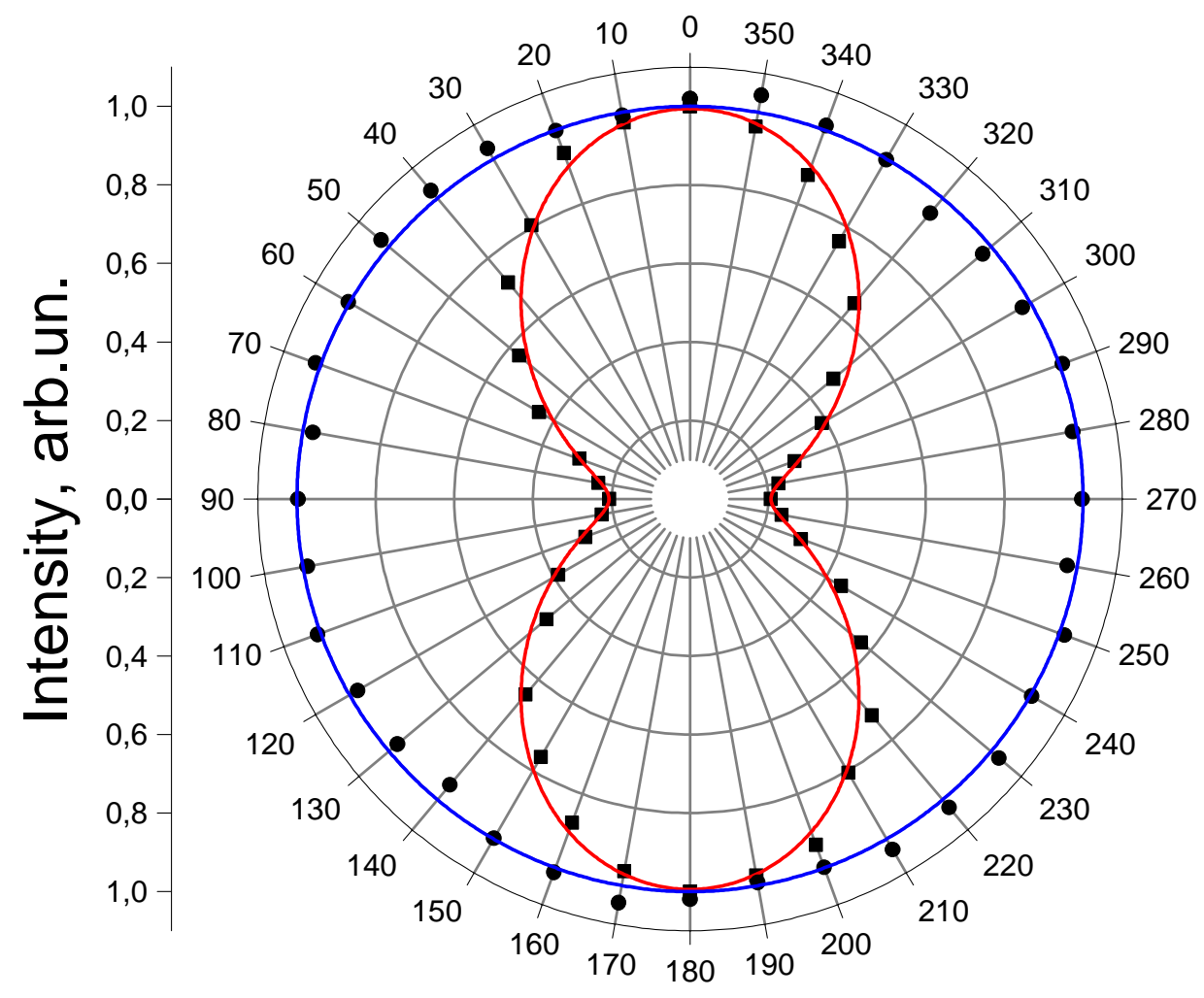

Figure S4. Dependencies of light intensity passed through a Glan-prism polarizer on angle $\theta$ between vertical line and the polarizer axis; the dependencies were measured for the cases when a quarter-wave phase plate (see Figure S1) with the axis angle of $45^{\circ}$ in respect to vertical line was placed in front of the polarizer (circles) and without this phase plate (squares).

The dependence of the polarizer transmittance registered without the quarter-wave phase plate has been fitted well (red line) with the following equation:

$$
I(\theta)=I_{U}+I_{V} \cdot \cos ^{2}(\theta) .
$$

This dependence shows that the light is either elliptically polarized or partially linearly polarized in vertical direction. Additionally, a quarter-wave phase plate with the axis at the angle of $45^{\circ}$ in respect to vertical line was placed in front of the polarizer as it is shown in Figure S1. The dependence of the polarizer transmission measured with this plate can be well fitted with a circle (blue line). This evidences the absence of circularly of elliptically polarized light. Hence, the output radiation is exactly partially linearly polarized with $I_{U}$ and $I_{V}$ being the intensities of unpolarized and vertically linearly polarized components. Their values were estimated to be 0.20 and 0.79 arb.un., respectively. The dichroic ratio is 5 . 


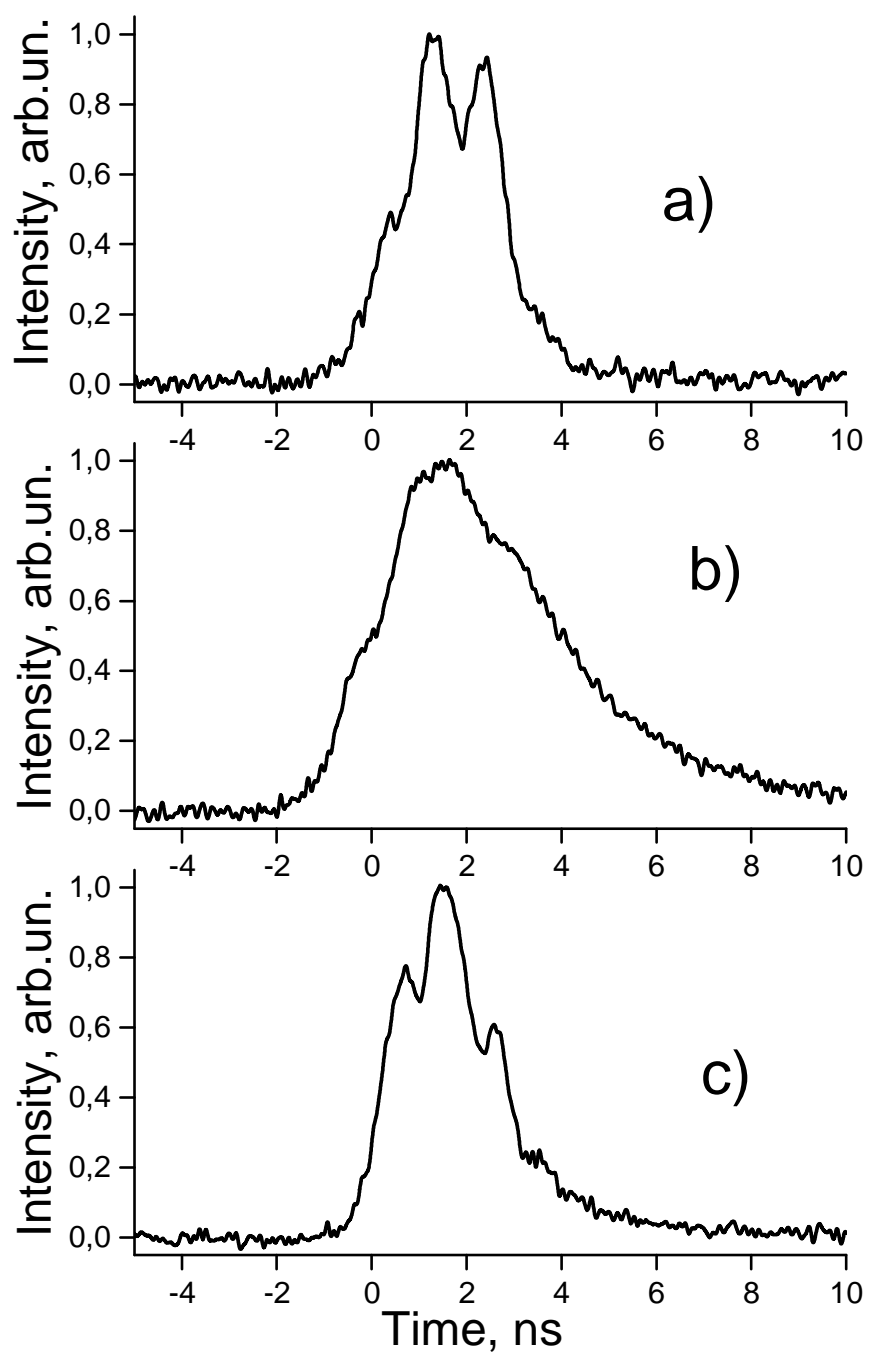

Figure S5. Oscilloscope traces of a pump pulse (a), of an emission pulse below the lasing threshold (b), and of a lasing pulse (c). The modulation of the pump pulse with a period of ca. $1 \mathrm{~ns}$ is explained with beating of modes in a multimode pump laser. Below the lasing threshold (Figure S5b) spontaneous emission with a lifetime of the nanosecond order made the emitted pulse smoother and longer than the pump pulse; the pulse modulation is almost unnoticeable. As soon as the random lasing started, the emitted pulse began to reproduce the shape of the pump pulse having only the sharper leading edge than that of the pump pulse (see Figure S5c). 

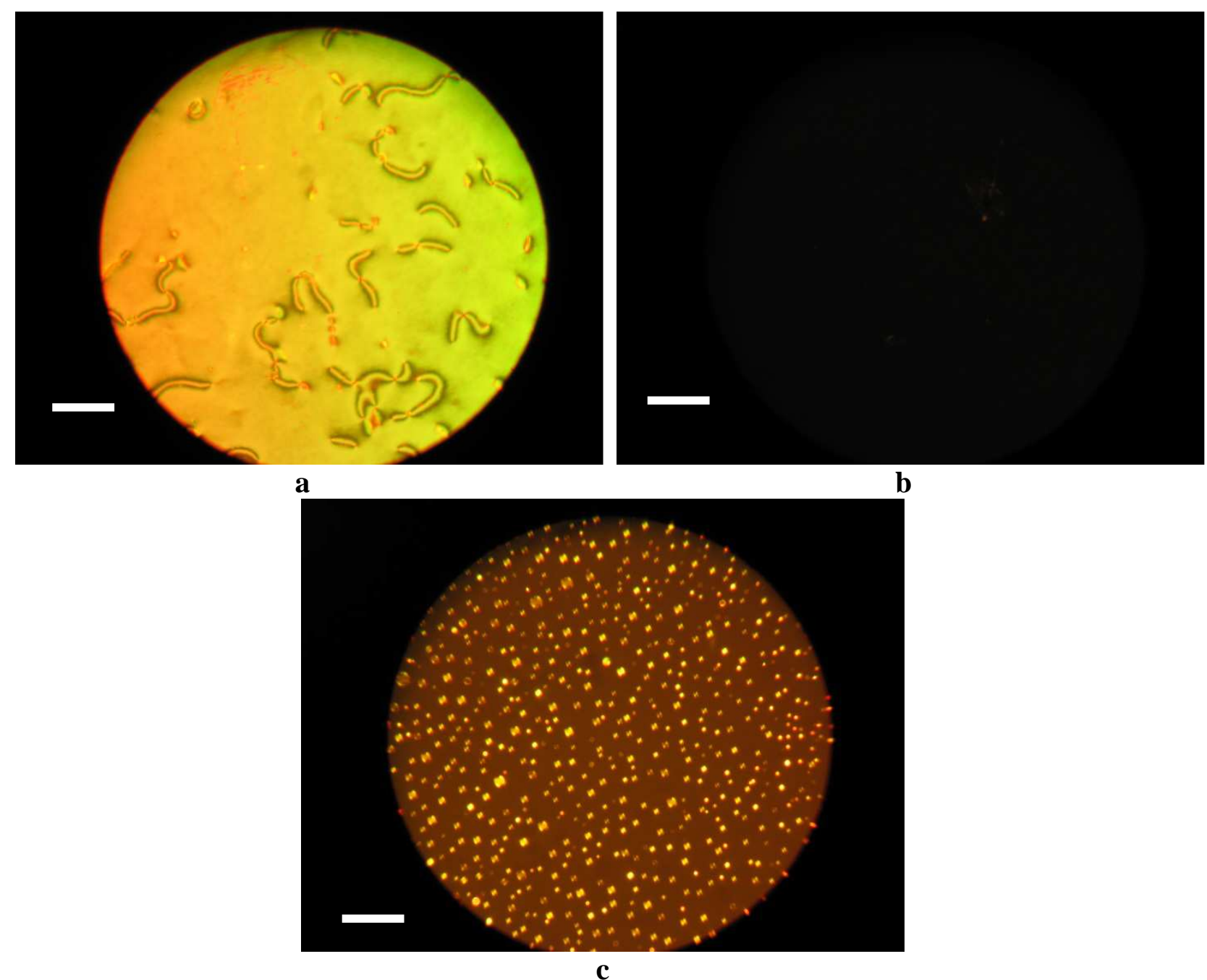

Figure S6. Polarizing optical microscope images of the 5CB:6DABU mixture (a) before, (b) after UV irradiation $\left(380 \mathrm{~nm}, \sim 20 \mathrm{~mW} / \mathrm{cm}^{2}, 10 \mathrm{~s}\right)$, and (c) after thermal relaxation at room temperature for 18 days. Scale bar is $100 \mu \mathrm{m}$. 


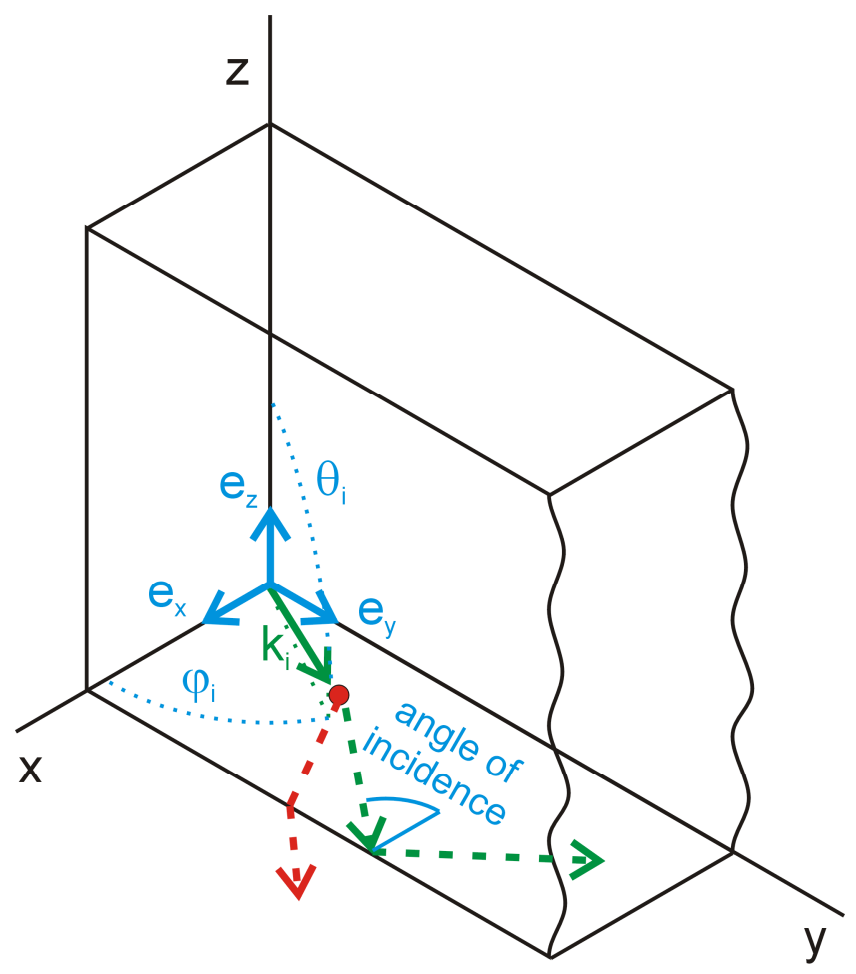

Figure S7. Schematic representation of the coordinate system and simplified illustration of the losses due to transmittance through sides of the film; light traveling along the film (green solid arrow) scatters producing scattered rays (dashed lines) so that for one of the rays (green line) the angle of incidence to the film surface is higher than the angle of the total internal reflection and this ray propagates further, while for the second ray (red line) the incidence angle is small, the refraction occurs and the ray leaves the film.
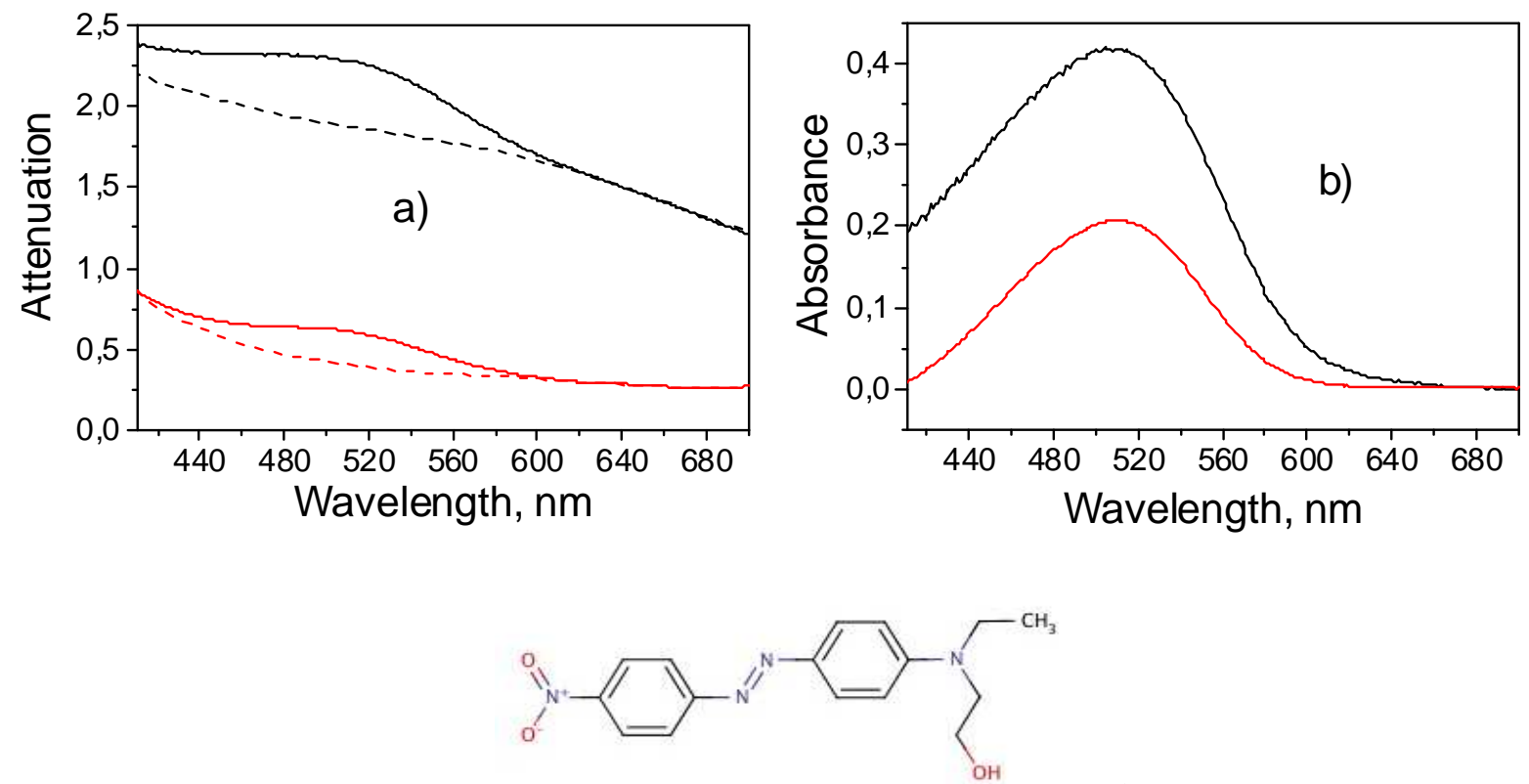

c)

Figure S8. a) Polarized attenuation spectra of a porous PP film filled with nematic 5CB LC doped (solid lines) with dichroic azobenzene dye (Disperse Red 1, 0.3 wt.\%) and undoped (dashed line); spectra were measured parallel (black lines) and perpendicular (red lines) to the LC alignment direction (or the stretching direction of the PP film) with an UV-visible 
spectrophotometer (UNICAM UV-500); b) absorbance spectra of Disperse Red 1 (DR1) calculated with subtraction of the attenuation spectra; c) chemical structure of DR1.

The order parameter was calculated according to the following formula

$$
S_{d}=\frac{A_{p}-A_{s}}{A_{p}+2 A_{s}}
$$

where $A_{p}$ and $A_{s}$ are absorbances of the DR1 in the sample for radiation polarized parallel to the LC director and orthogonal to it, respectively.

In order to determine the absorbance of the DR1 attenuation of two samples was detected (Figure S8a). The first sample was filled with the nematic LC doped with DR1 (solid lines), while the second was filled with the undoped LC (dashed lines). Subtraction of these spectra allowed us for excluding of light scattering, Fresnel losses, vignetting etc and resulted in absorption spectra of DR1 presented in Figure S8b. Values of $A_{p}$ and $A_{s}$ were equal to be 0.420 and 0.207 for the wavelength of $510 \mathrm{~nm}$ which corresponds to the absorption maximum. The order parameter was calculated with Eq.(S2) to be ca.0.3.

\section{Equations used for theoretical analysis}

Figure S7 presents the coordinate system used the analysis. The sample film was assumed to be located in the yz-plane, while the LC director was oriented along the z-axis. The inclination angle $\theta_{i}$ and azimuth angle $\varphi_{i}$ determined the direction of the wave vector of incident light $k_{i}$, while the angles $\theta_{s}$ and $\varphi_{s}$ determined the wave vector of scattered light $k_{s}$. The ordinary (light polarization is orthogonal to the plane formed by $k_{i, s}$ and the z-axis) and the extraordinary (light polarization is parallel to the plane) polarizations of incident and scattered waves were considered. It should be noted that such orientation of polarization vectors differs from that used in Ref., ${ }^{35-37}$ where vector orientation with respect to the scattering plane was used. The following equations were obtained for differential cross-sections $\frac{d \sigma_{\alpha \beta}}{d \Omega}$ of scattering of ordinary $(\beta=o)$ or extraordinary wave $(\beta=e)$ into ordinary $(\alpha=o)$ or extraordinary $(\alpha=e)$

$$
\frac{d \sigma_{o o}}{d \Omega}=k^{4} R^{6} f^{2} \cdot\left(\frac{2 n_{o}^{2}+n_{e}{ }^{2}-\left(n_{e}{ }^{2}-n_{o}{ }^{2}\right) S_{d}}{3 n_{m}{ }^{2}}-1\right)^{2} \cos ^{2}\left(\varphi_{s}-\varphi_{i}\right)
$$




$$
\begin{aligned}
& \frac{d \sigma_{o e}}{d \Omega}=k^{4} R^{6} f^{2} \cdot\left(\frac{2 n_{o}^{2}+n_{e}{ }^{2}-\left(n_{e}{ }^{2}-n_{o}{ }^{2}\right) S_{d}}{3 n_{m}{ }^{2}}-1\right)^{2} \cos ^{2} \theta_{i} \sin ^{2}\left(\varphi_{s}-\varphi_{i}\right) \\
& \frac{d \sigma_{e o}}{d \Omega}=k^{4} R^{6} f^{2} \cdot\left(\frac{2 n_{o}^{2}+n_{e}{ }^{2}-\left(n_{e}{ }^{2}-n_{o}^{2}\right) S_{d}}{3 n_{m}{ }^{2}}-1\right)^{2} \cos ^{2} \theta_{s} \sin ^{2}\left(\varphi_{i}-\varphi_{s}\right), \\
& \frac{d \sigma_{e e}}{d \Omega}=k^{4} R^{6} f^{2} \cdot\left(\begin{array}{l}
\left(\frac{2 n_{o}{ }^{2}+n_{e}{ }^{2}-\left(n_{e}{ }^{2}-n_{o}{ }^{2}\right) S_{d}}{3 n_{m}{ }^{2}}-1\right) \cos \theta_{i} \cos \theta_{s} \cos \left(\varphi_{s}-\varphi_{i}\right)+ \\
+\left(\frac{2 n_{o}{ }^{2}+n_{e}{ }^{2}}{3 n_{m}{ }^{2}}-1\right) \sin \theta_{s} \sin \theta_{i}
\end{array}\right)^{2}, \\
& f=\frac{\sin (a R)-a R \cos (a R)}{(a R)^{3}}, \\
& a=k \sqrt{2\left(1-\cos \theta_{i} \cos \theta_{s}-\sin \theta_{i} \sin \theta_{s} \cos \left(\varphi_{i}-\varphi_{s}\right)\right)},
\end{aligned}
$$

where $R$ is the radius of a LC-droplet, $S_{d}$ is the order parameter, which includes ordering of individual LC molecules along the droplet direction, thermoinduced fluctuations of LC-director, as well as disorientation of directors of different droplets $\left(S_{d}=1\right.$ corresponds to complete ordering of all LC molecules in all droplets, $S_{d}=0$ corresponds to isotropic orientation of all LC molecules). The wave number $(k)$ can be expressed as:

$$
k=2 \pi n_{m} / \lambda,
$$

where $\lambda$ is the light wavelength in vacuum $(804 \mathrm{~nm})$ and $n_{m}$ is refractive index of surrounding material (1.49).

The scattering cross-section $\left(\sigma_{\alpha \beta}\left(\theta_{i}, \varphi_{i}\right)\right)$ can be obtained by simple integration of Eqs.(S3)-(S6) over the whole solid angle:

$$
\sigma_{\alpha \beta}\left(\theta_{i}, \varphi_{i}\right)=\int_{0}^{\pi} \int_{0}^{2 \pi} \frac{d \sigma}{d \Omega} d \varphi_{s} \sin \left(\theta_{s}\right) d \theta_{s} .
$$

The refractive indices $n_{o}$ and $n_{e}$ were taken to be 1.524 and 1.689. The order parameter was assumed to be 0.4 for the case of LC state. This value this value provides the ratio of scattering 
cross-sections $\left(\frac{\sigma_{o e}+\sigma_{e e}}{\sigma_{o o}+\sigma_{e o}}\right)$ for extraordinary and ordinary waves calculated according to Eq.(S10) for normal incidence to the film $\left(\theta_{i}=90^{\circ}, \varphi_{i}=0^{\circ}\right)$ equal to 4 , which corresponds to the experimentally registered dichroic ratio. Additionally, the order parameter was estimated on the base of absorbance measurements for DR1 dye added to LC to be 0.3 (see Figure S8). Mismatch of these values of the order parameter can be explained both with imperfect alignment of the dye in LC (additional disorientation exists) and with imperfectness of theoretical model, which should be compensated with a higher value of order parameter.

The values of cross-sections $\left(\left\langle\sigma_{\alpha \beta}\right\rangle\right)$ averaged over all input angles $\left(\theta_{i}\right.$ and $\left.\varphi_{i}\right)$ were calculated as follows

$$
\left\langle\sigma_{\alpha \beta}\right\rangle=\frac{1}{4 \pi} \int_{0}^{\pi} \int_{0}^{2 \pi} \sigma_{\alpha \beta}\left(\theta_{i}, \varphi_{i}\right) d \varphi_{i} \sin \left(\theta_{i}\right) d \theta_{i}
$$

The condition for total internal reflection is

$$
1-\sin ^{2}\left(\theta_{s}\right) \cos ^{2}\left(\varphi_{s}\right)>\frac{1}{n_{m}^{2}}
$$

